\title{
Escuela urbana y reconfiguración de identidades en la juventud rural*
}

Urban School and Reconfiguration of Identities in Rural Youth

Escola urbana e reconfiguração da identidade na juventude rural

\section{José Eduardo Cifuentes-Garzón** iD orcid.org/0000-0002-5602-957X}

Para citar este artículo: Cifuentes-Garzón, J. (2021). Escuela urbana y reconfiguración de identidades en la juventud rural. Revista Colombiana de Educación, 1(82), 131-150. https://doi.org/ 10.17227/rce.num8210579

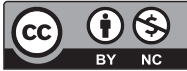

* Artículo derivado de la tesis doctoral "Escuela y construcción de identidades en jóvenes rurales: un abordaje desde las creencias y valores de los estudiantes de educación media del municipio de Villagómez Cundinamarca-Colombia".

** Doctor en Educación y Sociedad. Con Estudios en Alta Investigación Posdoctoral en Educación, Ciencias Sociales e Interculturalidad. Rector de la Secretaría de Educación de Cundinamarca. 


\section{Resumen}

El artículo de investigación presenta las creencias y los valores subyacentes en las juventudes de un contexto rural y cómo la escuela influye en la reconfiguración de sus identidades, a fin de reconocerlos como actores sociales de desarrollo en sus comunidades. Se abordó un enfoque cualitativo, basado en la metodología etnográfica, desde una perspectiva hermenéutica y de doble reflexividad. Para recabar la información, se implementó la encuesta, los grupos de enfoque, la observación participante, el taller investigativo, la entrevista semiestructurada y, en profundidad, el diario de campo con la participación de 62 estudiantes y 10 profesores del nivel de educación media técnica. De acuerdo con los hallazgos, los jóvenes rurales reconfiguran sus identidades en el escenario escolar urbano, producto de la reflexividad de sus creencias, los valores y la búsqueda de reconocimiento y posicionamiento en escenarios sociales. Se apreció cómo las creencias (ideologías, religiosidad y tradiciones) y los valores (hábitos de hogar, principios y vínculos de unión) inciden en la configuración de las identidades (reflejadas en documentos, características propias y la otredad) de los jóvenes rurales.

\section{Palabras clave}

creencia; escuela; identidad; joven rural; valores
Keywords

belief; school; identity; rural youth; values

\begin{abstract}
The research article presents the beliefs and values underlying youth in a rural context and how schools influence the reconfiguration of their identities, in order to recognize them as social development actors in their communities. A qualitative approach, based on ethnographic methodology, was approached from a hermeneutical and double reflexivity perspective. To collect the information, the survey, focus groups, participant observation, research workshop, semi-structured and in-depth interview and field diary with the participation of 62 students and 10 professors of the technical secondary education level, were implemented. According to the findings, rural youth reconfigure their identities in the urban school setting, product of the reflexivity of their beliefs, values and the search for recognition and positioning in social settings. It was appreciated how the beliefs (ideologies, religiosity and traditions) and values (household habits, principles and bonds of union) affect the configuration of the identities (reflected in documents, own characteristics and otherness) of rural youth.
\end{abstract}

\section{Resumo}

O artigo de pesquisa apresenta as crenças e valores dos jovens no contexto rural e como a escola influencia a reconfiguração de suas identidades para reconhecê-los como atores do desenvolvimento social em suas comunidades. Utilizou-se uma abordagem qualitativa, baseada na metodologia etnográfica, sob uma perspectiva hermenêutica e de dupla reflexividade. Para coletar as informações, foram implementados a pesquisa, grupos focais, observação participante, oficina de pesquisa, entrevista semi-estruturada e, especialmente, o diário de campo com a participação de 62 alunos e 10 professores do Ensino Médio técnico. De acordo com as descobertas, os jovens que moram em zonas rurais reconfiguram suas identidades no ambiente escolar urbano, produto da reflexividade de suas crenças, valores e a busca pelo reconhecimento e o posicionamento nos ambientes sociais. Observou-se como as crenças (ideologias, religiosidade e tradições) e os valores (hábitos domésticos, princípios e vínculos de união) afetam a configuração das identidades (refletidas em documentos, características próprias e alteridade) da juventude rural.

\section{Palavras-chave}

crença; escola; identidade; juventude rural; valores 


\section{Introducción}

En los diferentes niveles educativos los niños, las niñas y los jóvenes transitan inadvertidos. La escuela sigue distante de los contextos, las condiciones y las realidades de los estudiantes que afectan las formas de actuar en los distintos escenarios personales, familiares y comunitarios. En este sentido, los procesos de construcción de identidades en las juventudes rurales y del sector urbano son invisibilizados y poco se ha indagado al respecto. De acuerdo con Meseguer (2012, p. 122), "La invisibilidad de la juventud rural ha persistido porque se la sigue considerando más que como actor social, como una población vulnerable a la que hay que proteger e integrar y sobre la que descansa la promesa del desarrollo agrícola". Además, los jóvenes colombianos están todavía en una situación de aislamiento caracterizada por "pocas posibilidades de participación política, un futuro de desempleo o subempleo, una educación de baja calidad que no entusiasma y que no asegura ni empleo ni movilidad social, y una sociedad sin un modelo claro de futuro" (Parra, 1986, p. 81). Por tal razón, urge la necesidad de generar espacios para los jóvenes, a fin de escucharlos y poder redireccionar los procesos educativos. En esta perspectiva, Alvarado, refiriéndose a los jóvenes, considera "necesario mover los límites categoriales desde los cuales, tradicionalmente, los habíamos asumido como objetos pasivos y dependientes, para reconstruir con ellos las coordenadas sociales y simbólicas que nos permitieran comprenderlos como sujetos activos de discurso y acción" (2014, p. 40).

En consecuencia, se requiere investigar el papel de la escuela en la configuración de los saberes y las creencias en cuanto a la ruralidad, la territorialidad, la cultura campesina y los valores arraigados en los jóvenes, con el propósito de reconocerlos como agentes protagonistas del desarrollo en sus comunidades y capaces de valorar sus identidades. Por tanto, se implementó el presente proyecto de investigación sobre la construcción de identidades en las juventudes en contextos rurales y la manera como la escuela interviene en la permanencia en los territorios o en la emigración a la ciudad de los jóvenes. Por ello, el problema de investigación fue guiado por el interrogante: ¿Cómo afecta la escuela urbana la construcción de identidades, asociadas a las creencias y los valores de los jóvenes rurales de educación media del municipio de Villagómez, en Cundinamarca (Colombia)?

Con el fin de responder esta pregunta, el objetivo general planteado fue: comprender la incidencia de la escuela urbana en la construcción de la identidad del joven rural, a través del análisis etnográfico hermenéutico y de doble reflexividad de las creencias y valores de los estudiantes de educación media de una institución pública del municipio de Villagómez en el departamento de Cundinamarca Colombia, a fin de visibilizarlos y empoderarlos como actores sociales en el desarrollo de sus comunidades.

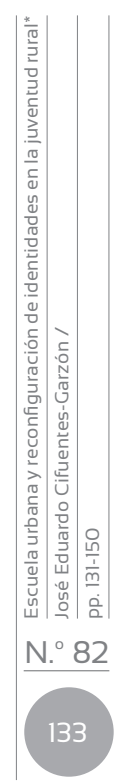


El artículo reporta los hallazgos de la investigación relacionados con el objetivo específico reportar las nociones de creencias y valores subyacentes en la juventud rural y la incidencia de la escuela urbana en la reconfiguración de sus identidades.

En esta investigación, la identidad se concibe como "un proceso cognitivo, individual y social, que permite al sujeto darse cuenta de su propia existencia y tomar conciencia de sí mismo. Para que estos procesos se produzcan, el individuo debe percibirse diferente del Otro" (Torres, 2019, p. 5). De igual modo, la escuela se concibe como una microsociedad, en la cual convergen diversos actores con características propias, generadores de situaciones y relaciones complejas. Por tanto, las instituciones escolares cumplen una función trascendental en la construcción de la identidad de los jóvenes rurales, a través del cúmulo de interacciones con el currículo, los estudiantes, los docentes y demás sujetos del escenario educativo, al consolidar valores y creencias que habilitan a los estudiantes para actuar en sociedad, en estas realidades en las cuales lo foráneo interfiere en los pensamientos, las decisiones y los procederes de la juventud.

En relación con las creencias, es importante el papel de la religión en la parte constitutiva de la formación humana. En este sentido, "La religión individualizada en cada uno de nosotros como un sistema de creencias brinda distintas pautas de comportamiento, maneras de entender el mundo, a los demás y a nosotros mismos, de este modo ofrece un propósito a nuestro proyecto vital" (Oñate et ál., 2018, p. 59). Es decir, los credos religiosos desde sus principios asumen los problemas y circunstancias del ser humano, lo cual incide en la consolidación de rasgos identitarios. La religión es una fuente de identidad, da cohesión social, ofrece motivaciones y creencias que afectan los ámbitos moral, político y social de la persona (Estrada, 2011).

En cuanto a los valores, Etxeberria (2011), hace aproximaciones a la identidad desde la perspectiva de la ética, al señalar cómo cada individuo participa en la configuración de la identidad humana, dada por la libertad y la racionalidad, aspectos que constituyen a la persona en sujeto de dignidad. En este sentido, "Las identidades se construyen en un doble proceso de socialización (interiorización de normas y valores) y de subjetivación (emancipación y elaboración de normas y valores propios), sobre todo al convivir con otros (sociabilidad)" (Weiss, 2012, p. 141), dependiendo de factores como "los valores, normas y conocimientos adquiridos en su ámbito cultural" (Alzate et ál., 2018, p. 99). Por consiguiente, las prácticas culturales del medio rural determinan las posibilidades de concebir el mundo y sus realidades, desde los procesos reflexivos provocados en el ámbito escolar, en la búsqueda de visibilizar a los jóvenes como sujetos sociales que suelen encontrar "dificultades en organizar el futuro, en definir 
el deseo, en construir metas vitales, proyectos de vida, que establezcan su acción, que desaten una actividad con sentido, en lo que se refiere a la felicidad" (Parra, 2008, p. 302)

\section{Metodología}

La investigación se desarrolló en una institución educativa pública del municipio de Villagómez en Cundinamarca (Colombia). Para el 2018, esta institución cuenta con 425 estudiantes, 224 en la sede principal y los demás en sus 12 sedes rurales, 10 docentes en la sede principal y 15 en las rurales. Los participantes de la investigación fueron 32 estudiantes del grado décimo y 30 de undécimo. Las condiciones de vida de dichos jóvenes los ubican en los niveles socioeconómicos bajo y medio, y la principal actividad económica de sus padres es la agricultura. Para el desarrollo del ejercicio investigativo se trabajó solo con los estudiantes de educación media procedentes del campo y cercanos a finalizar su ciclo de educación, a fin de analizar la manera como estos jóvenes son vinculados en las dinámicas de los colegios urbanos y cómo la escuela contribuye en la reconfiguración de sus identidades. Concernía abordar las perspectivas respecto a la identidad en el presente y hacia el futuro, vista desde los valores y las creencias.

El estudio fue desarrollado siguiendo un enfoque cualitativo, el cual permitió develar cómo los jóvenes construyen sus identidades desde la interacción con el contexto escolar, al visibilizar diversas problemáticas y generar reflexividades para transformarlas, a partir de las mismas perspectivas y los encuentros etnográficos con los jóvenes rurales. Para tratar el estudio de las juventudes rurales a fin de empoderarlas en el desarrollo del campo, resultó apropiado optar por la etnografía, entendida como "el arte y la ciencia de describir a un grupo: sus instituciones, comportamientos interpersonales, producciones materiales y creencias" (Angrosino, 2012, p. 35). La investigación abordó la metodología etnográfica, con una perspectiva hermenéutica y de doble reflexividad, a partir de entrevistas etnográficas, encuestas sociodemográficas, grupos de enfoque, cartografía social y observaciones participantes de habitus intra- e interculturales de los jóvenes rurales y la articulación de los discursos de identidad y las prácticas de interacción, recogidas en talleres investigativos y análisis documentales.

\section{Resultados}

Este apartado ilustra cómo los jóvenes rurales reconfiguran sus identidades en el escenario escolar urbano, producto de la misma reflexividad de sus creencias y valores: 


\section{Lo difícil de ser joven rural: "gente sufrida pero agradecida"1}

Las diferentes conversaciones realizadas con los jóvenes de educación media del municipio de Villagómez (Cundinamarca) evidencian el reconocimiento de sus valores, el aprecio por su territorio y las dificultades a las cuales se ven enfrentados. Con relación a la manera como son percibidos, los estudiantes manifiestan:

Los jóvenes rurales son de las veredas y sufren más para tener una educación. Como personas son más dedicadas al campo y llenas de valores en nuestro entorno. Son personas que se esfuerzan para salir adelante con trabajo, honestidad y dedicación. Aún tienen un poco de cultura y están más aislados de tantas problemáticas: drogas, violencia y vagancia. (Es, estudiantes 10 $0^{\circ}$. 24/11/2016) ${ }^{2}$

"Somos muchachos emprendedores, trabajadores, responsables, honestos, disciplinados, alegres y tímidos. Destacan que hay más cultura en los jóvenes rurales, mejor aprovechamiento en el tiempo libre y más esfuerzo en las labores diarias. En cuanto a las ventajas de ser joven rural declaran estar alejados de vicios, malas costumbres. Hay Tranquilidad, libertad, podemos cultivar nuestros propios alimentos y aprendemos más sobre el campo. Valoramos el estudio y más oportunidad laboral. Tenemos un medio ambiente sano, la facilidad de tener los productos para la alimentación, tranquilidad y programas sociales" (Gf. 1, 2, 3 y 4, estudiantes, 06/10/2016).

"Como buenas personas, somos gente sufrida pero agradecida de todo [...] Somos jóvenes trabajadores y entusiastas que valoramos lo que tenemos" (Es, estudiantes, 23/11/2016)

De acuerdo con lo anterior, los estudiantes conectan la condición de ser joven rural con su territorio y las labores propias de este entorno. El trabajo en el campo, la interacción con el contexto y las relaciones con sus pares, generan una visión de sí mismo, permeada por valores propios, los cuales hacen distintos a los demás grupos. Por eso, en la escuela existe mayor empatía entre los docentes y los estudiantes provenientes del sector rural, quienes más dóciles y obedientes. Al respecto, Bustos, señala: "Se suele escuchar que es un alumnado más respetuoso con sus iguales y con el profesorado, así como dotado de una mejor conducta social que le lleva a mostrar pocos comportamientos disruptivos" (2012, p. 75). Lo dicho hasta

1 De aquí en adelante, los subtítulos de este apartado corresponden a expresiones propias de los jóvenes, las cuales emergieron en el transcurso de la investigación.

2 Lo citado textualmente tendrá la siguiente nomenclatura: Análisis documental (Ad) Diario de campo (Dc), Rutina de pensamiento (Rp), Cartografía social (Cs), Grupo focal (Gf), Entrevista semiestructurada (Es), Entrevista en profundidad (Ep), Taller investigativo (Ti), Notas de campo (Nc), seguido de la numeración, el código del estudiante o profesor participante y la fecha del registro. 
aquí, supone ver a los jóvenes rurales con características inherentes a su lugar de procedencia, es decir, el hecho de interactuar con su contexto, participar de las labores del campo y el estar alejados de los medios masivos de comunicación, posibilita el desarrollo de actitudes más cercanas a la disciplina de aula deseada por los profesores. Por lo general, sus normas están centradas en el porte correcto de uniforme, el silencio en clase y el cumplimiento de tareas, procuran estudiantes menos cuestionadores y adaptados al régimen establecido.

En relación con lo dicho, un profesor de educación media de la institución donde se desarrolló la investigación hace la diferencia entre jóvenes del campo y del pueblo en los siguientes términos:

La institución está en el pueblo, que es la zona urbana de alguna manera, considerando esto se propone dos poblaciones. Por un lado, los jóvenes que realmente viven en la zona rural, los estudiantes que saben de las labores del campo, de ordeñar y sembrar, ellos construyen una identidad más noble, humilde y respetuosa en todo sentido. Por otro lado, los jóvenes de la zona urbana construyen una identidad y unas costumbres muy diferentes, están más acostumbrados a las facilidades de la vida y de sus padres. (Nc, profesor 1, 12/01/2017)

Los mismos jóvenes proponen otras características de estos dos grupos:

El del sector urbano es más "desarrollado" o sabe más sobre tecnología, pero igual en el campo se vive la tranquilidad. Los jóvenes urbanos tienen posibilidades de más cosas, por ejemplo, campeonatos, concursos, en fin, y los del sector rural nunca los tienen en cuenta, siempre están abajo o no saben qué quieren ellos hacer. (Es, 23/11/2016).

El joven rural está más aislado de los vicios y tienen más espacio y tiempo de vagar y el joven urbano tiende a poseer más accesibilidad a drogas, alcohol, fiestas, etc. (Es, 24/11/2016)

Con relación a los aprendizajes, señalan

Ha sido difícil, por la lejanía al colegio en el sector urbano, la cual me obliga a madrugar y a caminar extensas jornadas diarias. En aprendizaje los jóvenes rurales tienen más conocimientos. En deportes los jóvenes urbanos tienen ventajas frente a los rurales". (Es, estudiantes, 24/11/2016)

Además, agrega uno de los jóvenes:

Yo creo que en este sector hay tanto ventajas como desventajas, una de las principales desventajas que yo le he visto a la mayor parte de la zona rural es la poca conectividad y falta de información. Yo creo que la gente del campo es más amable, hay más respeto hacia las demás personas. (Ep, estudiante 1, 08/12/2016) 
El estudiante continúa su discurso de la siguiente manera:

Es todo más las costumbres, porque la educación que recibe uno en el campo y las enseñanzas de los padres y de las personas con las que vive, va a ser muy distinto a las que recibe uno en la ciudad. Entonces el comportamiento va a ser distinto, quizás la moda, la forma de hablar, de vestirse, van a ser cosas que para expresarlas que uno viene del campo, puede ser un factor de discriminación por parte de los jóvenes que han sido criados en el entorno urbano. (Ep, estudiante 1, 08/12/2016)

En este sentido, "El vestuario, la música, el acceso a ciertos objetos emblemáticos, constituyen hoy una de las más importantes mediaciones para la construcción identitaria de los jóvenes" (Reguillo, 2000, p. 27).

Por otra parte, "la juventud es una noción dinámica, sociohistórica y culturalmente construida, que es siempre situada y relacional" (Vommaro, 2016, p. 18). Es decir, los jóvenes rurales reconfiguran sus identidades según su contexto particular. Para ellos, las limitaciones al acceso a expresiones culturales, artísticas y deportivas, los ponen en desventaja frente a los jóvenes urbanos. Sin embargo, agencian oportunidades, destacan las bondades de vivir en el campo y reafirman su arraigo al territorio, aunque al finalizar su trayectoria escolar decidan emigrar a la ciudad.

En cuanto a lo difícil de ser joven rural, los estudiantes indican:

Lo complicado es la dificultad para trasladarse hacia un centro educativo o centros de salud y que los que se creen habitantes de la ciudad, traten de vernos como esclavos de la tierra. En el lugar donde uno vive no hay los medios para la adecuada educación y desarrollo como persona, es decir, falta de bibliotecas en las veredas, falta de centros de integración ciudadana, por estas razones es necesario trasladarse del área rural a la urbana, pero es complicado por la falta de transporte. Otro aspecto importante, es la falta de interés de las autoridades del municipio por nosotros. (Es, estudiantes, 24/11/2016)

Llama poderosamente la atención dos expresiones: "tratan de vernos como esclavos de la tierra" y "la falta de interés de las autoridades del municipio por nosotros". La primera refleja el sentimiento de humillación de estos estudiantes cuando interactúan con otros jóvenes de la ciudad. La segunda, refleja el descuido sistemático de la política pública por la juventud rural. Según Paulín: "Para los jóvenes, al involucrarse en las actividades relacionales cotidianas significándolas y significándose con voz propia, puede construir un espacio social habilitante de sus identidades" (2016, p. 150). Es decir, el reconocimiento de estas realidades es una oportunidad de potenciar acciones enfocadas a la transformación del espacio y de las relaciones sociales, en el sentido de cerrar las brechas entre los habitantes del sector rural y del urbano. 


\section{"La identidad es como un tatuaje... nos marca para siempre"}

Los jóvenes rurales relacionan sus identidades con:

Aquel joven que ha nacido y vivido en el campo, su lugar de residencia está dado en un contexto diferente al urbano. Comprende también las actitudes y virtudes que posee un individuo, son aquellas características que definen a una persona, sus valores, raíces. Es decir, la identidad es como un tatuaje, que nos marca para siempre. (Gf3, estudiantes, 06/10/2016)

Somos muy tímidos y por llamarlo así "huevones" al dejarnos maltratar de otra persona, somos muy honestos y callados. (Es, estudiantes, 23/11/2016)

Quizás en algunos casos a los muchachos les da pena decir que son del campo, porque de pronto tienen miedo a que los discriminen, porque son del campo y esto nada más cuando van a sitios en los que sabe que la mayoría de los jóvenes son del contexto urbano, a uno le da miedo decir que es del campo por temor a que lo rechacen o que no lo acepten. (Ep, estudiante 2, 08/12/2016)

En esta perspectiva, Jurado y Tobasura (2012) plantean la identidad desde lo cultural, vinculando los rasgos propios de una comunidad, las formas locales de pensar, ser y actuar, además de las tensiones propias del territorio y el arraigo a la vida rural. Es decir, la identidad de la población joven que habita los territorios rurales, está relacionada con las maneras de construir sus proyectos de vida, desde las posibilidades y dificultades en el despliegue de potencialidades, oportunidades del medio rural, capacidades de participación en la vereda y en el municipio. De esta manera se configura la identidad cultural, la cual se reconfigura en las interacciones con los escenarios comunitarios, entre ellos, el escolar.

Al preguntarles a los estudiantes si la escuela y los maestros inciden en la construcción de las identidades de los jóvenes rurales, ellos dijeron:

Sí, porque la institución tiene como base la igualdad, promueve la multiculturalidad, integra a sus estudiantes en las actividades colectivas que se realizan sin importar de qué índole son dichas actividades deportivas, religiosas, culturales etc. (Gf3, 06/10/2016)

A mí me enseñaron que así fuera del campo, que fuera hijo de gente campesina, éramos iguales a los del pueblo y que a los hijos de cualquier personalidad que sea, como sea, somos iguales. La condición humana que tenemos no es que, porque seamos blancos, negros, del pueblo o del campo, pues somos iguales, entonces yo creo que la escuela siempre contribuye a que uno se sienta como valorado y que le dé la importancia al contexto en el que vive. (Ep, estudiante 1, 08/12/2016) 
Con relación a la contribución de los docentes en la construcción de la identidad del joven rural, algunos estudiantes manifestaron:

Los maestros no contribuyen porque se enfocan en los jóvenes urbanos y no somos tenidos en cuenta. Sin embargo, la contribución es a partir de las experiencias, con consejos y con todo el conocimiento aprendido de ellos. (Es, estudiantes, 24/11/2016)

De acuerdo con Parra et ál: "La influencia del maestro en niños y jóvenes es fundamental y decisiva [...] los estudiantes tienen en el maestro a un adulto significativo en quien depositan su credibilidad y de quien aprenden no sólo conocimientos sino también relaciones, significaciones, sentidos" (2006, p. 67).

Por su parte, una profesora caracteriza a los jóvenes del municipio de Villagómez de la siguiente manera:

La niñez y juventud del sector rural de nuestro municipio, son personas que tienen una cultura basada en valores y buenas costumbres, son jóvenes emprendedores y saben enfrentar en su vida cotidiana cualquier trabajo, además de ser personas muy humildes y que tienen muchas dificultades de tener oportunidades de superación y de poder cumplir sus sueños por falta de recursos y apoyo por parte del Gobierno, siguen su vida y se dedican a trabajos humildes para poder sobrevivir. (Nc, profesora 2, 12/01/2017)

Conviene subrayar la capacidad de agencia y resistencia de los jóvenes rurales, porque a pesar de las condiciones de vulnerabilidad en este sector, procuran gestionar su sostenibilidad económica en los oficios de la agricultura. La tenacidad es una cualidad que se destaca en estos estudiantes, al asumir el reto de abandonar su territorio y emprender la búsqueda de nuevas posibilidades de vida en la ciudad, como protesta ante el abandono de las políticas públicas frente a las necesidades de las comunidades rurales.

Según Chaves (2010), los diversos estudios sobre juventud en la institución educativa muestran a la escuela como un lugar importante para los jóvenes, donde ocurre la sociabilidad juvenil, al ser un espacio de interacción entre amigos. Por tal razón, al dar una mirada integral a los jóvenes en los contextos escolares, resulta oportuno fijarse en las relaciones sociales, el manejo del poder, la subordinación y el dominio. La escuela como institución contribuye en la construcción de individuos heterónomos y muy poco autónomos. La construcción del individuo autónomo implica racionalidad y libertad para actuar con responsabilidad por convicción, porque los jóvenes están generalmente en posiciones de subordinación. Es así como la escuela urbana se convierte en escenario para los jóvenes rurales de interacción y resignificación de identidades con los sujetos actuantes, en la búsqueda de posicionamiento y reconocimiento en la sociedad. 


\section{De la escuela rural a la urbana: "nos tocaba como perro regañado"}

Un hecho importante para los jóvenes rurales ha sido el cambio de la escuela rural a la escuela urbana. En la mayoría de los municipios del departamento de Cundinamarca los niños estudian los niveles de preescolar y primaria en las escuelas de cada vereda con el modelo educativo Escuela Nueva y continúan la básica secundaria en instituciones ubicadas en el sector urbano. Al respecto, los jóvenes entrevistados comentaron lo siguiente:

Yo llegué allá al colegio con temor porque solo conocía a los compañeros con los que íbamos de acá del campo. Además, no conocía a los docentes, también tenía miedo de la metodología de la enseñanza porque yo sabía que iba a cambiar, por allá iba a ser otro método de enseñanza y no lo conocía, no sabía si me iba a acoplar a ese método. (Ep, estudiante 2, 08/12/2016)

"El cambio más grande fue que nosotros allá en la escuela, uno trabajaba y el profesor era como un guía que le iba explicando lo que uno no entendiera en los libros, mientras que allá en el colegio, era distinto porque aparte de que allá era un solo profesor el que orientaba todas las áreas, mientras que, en el colegio del pueblo, generalmente un profesor orientaba dos, tres, cuatro áreas. Eran varios profesores y la metodología de enseñanza era distinta porque ya uno no trabajaba con una guía, sino era lo que el profesor llevara preparado para su clase. (Ep, estudiante 3, 08/12/2016)

Una de las diferencias de la escuela rural en relación con la urbana es que, en esta última, "el enfoque ha estado aún más focalizado sobre el contenido de la materia a enseñar; o sea, los docentes enseñan materias, no a alumnos" (Anderson, 2011, p. 118). La falta de articulación entre ambas es una causa de la repitencia escolar. Es decir, la desconexión entre la escuela primaria y la secundaria, al no tener en cuenta los contextos particulares donde se desenvuelven los estudiantes, conduce al fracaso y a la deserción escolar, debido en gran parte al desconocimiento del profesorado de bachillerato de los métodos, estilos de aprendizaje, recursos y prácticas de aula con los cuales inician los estudiantes su formación escolar en el sector rural. Los sujetos actuantes de la escuela no tienen en cuenta la tensión ocasionada en la mayoría de los jóvenes que pasan de lo rural a lo urbano. Por consiguiente, estas situaciones complejas inciden en la manera como ellos se posesionan ante sus pares, y como acción de resistencia optan por abandonar sus estudios. Los jóvenes plantean esta situación así:

Entramos al colegio del pueblo, después de haber cursado noveno en el colegio del campo, dos perdieron décimo, uno siguió repitiendo el otro no siguió estudiando y yo me gradué con otro compañero. Al principio, yo como no conocía a nadie, casi solo me hablaba así 
con los que conocía, pero ya después en lo del proceso del estudio y así el aprendizaje, uno se va como relacionando con los demás compañeros y al final ya se conoce con todos y ya es como mejor el ambiente y amistad con todos los compañeros. (Ep, estudiante 4, 08/12/2016).

En el caso de los jóvenes rurales, al integrarse a las dinámicas de la escuela urbana con nuevos compañeros y profesores, establecen relaciones distintas, mediadas por aceptaciones, rechazos y transformaciones. Al respecto, un estudiante señala:

Yo en ocasiones sí notaba que los del pueblo eran como algo indiferentes con los que éramos del campo, pero no era algo tan notorio. Al principio las actividades las lideraban los del pueblo. En varias ocasiones, yo me acuerdo, los grupos de trabajo se hacían era los del pueblo y ellos eran los que sabían, a uno le tocaba "como perro regañado", ahí a un lado, esperando a ver qué grupo nos acogía. (Ep, estudiante $1,08 / 12 / 2016)$

A su vez, una profesora comenta los cambios de los estudiantes de esta manera:

Los niños en las escuelas rurales, son humildes, trabajadores colaboradores tanto en la casa como en la escuela, al momento de llegar al colegio, para algunos su cambio es notable puesto que se creen con libertad para realizar cosas que antes no podían hacer, se olvidan en dónde [sic] nacieron y sus costumbres las cambian por el entorno donde se encuentran. (Nc, profesora 3, 12/01/2017)

En el tránsito de la primaria rural a la educación básica y media en lo urbano suceden acontecimientos valiosos para la determinación de las identidades ante los cuales la escuela no puede permanecer indiferente. Por tal razón, en el marco de lo rural, la institución educativa tiene el compromiso de asumir como nuevo desafío, en los distintos niveles, ofrecer una educación al joven rural que le ayude insertarse en el campo laboral y en los procesos de desarrollo social de sus comunidades.

\section{"Que yo me acuerde" las creencias "eran temas que no se tocaban"}

Los jóvenes rurales del municipio de Villagómez Cundinamarca hacen referencia a las creencias y los valores de la siguiente manera:

[Las creencias] Es lo que uno ha aceptado y posee como ideas propias, son ciertos principios de los cuales uno no se desprende y los lleva consigo, ya sea porque nos los enseñaron o los hemos aprendido, por ejemplo, la religión. (Gf3, estudiantes, 06/10/2016) 
Es como el conjunto de ideas, valores y principios que muestran la existencia de un ser supremo, de Dios, pues que nosotros no estamos aquí y que podemos hacer lo que hagamos, sino que la moral nos dice que debemos hacer lo que esté bien. (Ep, estudiante 5, 08/12/2016)

En cuanto a las actitudes de los jóvenes hacia las creencias religiosas, los jóvenes comentan:

Que la mayoría de los muchachos son de la religión de sus padres, pero no son tan seguidores de ella. (Es, estudiantes, 23/11/2016)

Yo me acuerdo de que casi nunca, eran temas que no se tocaban, hablábamos de muchas otras cosas de deportes, de música, de cualquier otra cosa pero que yo me acuerde así, casi nunca hablábamos de la iglesia, de Dios, ni casi nada de eso. (Ep, estudiante 3, 08/12/2016)

Sin embargo, al preguntar a los jóvenes por la religión que profesan, la mayoría de ellos dijeron ser católicos. Por tanto, las creencias religiosas de los jóvenes rurales, inspiradas en la fe católica se manifiestan en los actos exteriores como la Eucaristía, el viacrucis, las procesiones de Semana Santa, las festividades marianas y la Navidad. Estos actos les permiten reflexionar sobre experiencias de vida, compartir con otros y fortalecer la unidad familiar. No obstante, algunos no participan en las celebraciones católicas, por pertenecer a religiones distintas, desinterés hacia estas celebraciones y por la presión de grupo de quienes ven en los actos religiosos algo pasado de moda y propio de los adultos mayores.

Con relación a la incidencia de la religión en la identidad de las personas, un estudiante comenta:

Yo creo que sí, porque si uno recibe lo que le dan los padres, de la religión, de la existencia de Dios y que Dios es el Ser supremo, eso como que la doctrina religiosa trae consigo unos principios, dicen que debe portarse bien, que uno no debe pecar, entonces es algo que los padres nos enseñan y que uno lo tiene presente. (Ep, estudiante 1, 08/12/2016).

En el discurso, los estudiantes afirman estar alejados de la religión, aunque dicen profesar el catolicismo y tienen creencias religiosas arraigadas, como lo manifiestan en las explicaciones de las cartografías sociales:

entonces vemos que nosotros tenemos como ejes morales a Dios y el campo como parte de nuestra naturaleza y como parte de Dios. De la misma manera de llegar a Dios, a la religión, utilizando la Biblia o de acuerdo con la religión de los hermanos que por lo general es la mayoría católica. Tenemos en cuenta los valores familiares, la responsabilidad, los esfuerzos." (Cs1, estudiantes 10, 10/09/2016)

Dios nos ayuda a tener fortaleza en los momentos difíciles, pedirle el apoyo, tranquilidad para cumplir lo que vayamos a hacer, por ejemplo, 
en los momentos difíciles nos olvidamos de él. (Cs2, estudiantes $10^{\circ}$, 10/09/2016)

porque si nosotros creemos en una religión, tenemos algo que nos caracteriza. Es algo en lo que confiamos y nos da vida, como una paz interior, nos da personalidad a nuestra vida. (Cs1, estudiantes $11^{\circ}$, $11 / 08 / 2016)$

Según los jóvenes rurales, los valores son"Actitudes que hemos adquirido y modifican nuestra identidad en nosotros y hacen parte de nuestra moral [...] Los valores como el trabajo, la responsabilidad y el esfuerzo son esenciales, además de la tolerancia y el cumplimiento, la cultura, la religión, el deporte y todo lo que adquirimos en el contexto rural, son esenciales y determinan nuestra identidad social. (Gf3, estudiantes, 06/10/2016)

nuestros padres nos enseñan algunos valores, estos nos sirven para la construcción de las identidades, porque nosotros si tenemos valores desde la casa, vamos a ser excelentes personas porque el buen ejemplo empieza desde casa. (Cs1, estudiantes 11ㅜ, 11/08/2016)

En relación con la pérdida de valores, un estudiante señala:

Yo creo que eso es verdad porque como decía antes... saludar a una persona mayor, es tener un respeto hacia él, si yo veo una persona que pasa a mi lado y no la saludo, eso es lo que se está viendo ahora, se puede decir que se está perdiendo el respeto, ya en cualquier circunstancia que alguien necesite y uno no lo ayuda... por eso se dice que se están perdiendo todos los valores, que no le ayudamos a las demás personas, no respetamos, inclusive ya casi ni buscamos trabajar, todo eso son cosas que se han perdido. (Ep, estudiante 4, 08/12/2016)

De acuerdo con lo anterior, la escuela rural debe contribuir en la conservación de los valores que han caracterizado a este sector de la población, a pesar de sus constantes transformaciones. Como lo sostiene Lozano: "La tradición intelectual de la sociología rural destaca entre las características principales del mundo rural su adscripción a los valores, pautas de comportamiento y creencias propias de la cultura tradicional, y el desarrollo de relaciones en un ámbito territorial restringido" (2012, p. 118). La tarea urgente de formar en valores para una sociedad en cambio está vigente en las instituciones educativas tanto rurales como urbanas, según las particularidades de sus habitantes.

\section{"Somos guerreros... podemos hacer de todo"}

En aras de privilegiar las voces de los jóvenes rurales, como lo hemos hecho, este último apartado presenta unas líneas de acción para empoderar a los jóvenes en sus contextos particulares, desde sus propias 
perspectivas. En este orden de ideas, a la pregunta ¿Qué debemos hacer para valorar la identidad del joven rural? Los participantes en la investigación respondieron:

Realizar más proyectos y actividades en nuestra zona, como somos del campo se debe realizar las actividades que se hacen aquí en el pueblo, deberían desplazarse hacia el campo donde todos podamos asistir y facilitarnos el transporte o los medios para llegar a ese sitio para poder participar en todas las actividades realizadas en el municipio. Yo pienso que se debería hacer más integración con los del pueblo. (Cs, estudiante 6, 11/08/2016)

Mantener en pie nuestros valores y creencias y tenerlas en cuenta para programas. Elaborar proyectos con los jóvenes del campo para mantener ese respeto por las personas y que nos respeten. (Cs, estudiante 7 , 10/09/2016)

Que se promueva la imagen del joven rural, que no trate de cambiarla con lujosos vestidos del pueblo, mejor dicho, que no cambiemos nuestra forma de vestir, de hablar, de expresarse, porque mucha gente lo critica a uno por ser campesino o la forma como uno es. (Cs, estudiante 8, 11/08/2016)

Que hagan más proyectos para que los del campo podamos participar más. Porque nosotros somos guerreros [...] podemos hacer de todo. (Gf 1 y 2 , estudiantes, $10^{\circ}-11^{\circ}, 10 / 09 / 2016$ )

Generar oferta educativa por parte del Gobierno y sus instituciones para garantizar el acceso a la educación superior y crear opciones de trabajo para los jóvenes que les genere un beneficio económico para su sustento y desarrollo. La vinculación educativa deberá estar enfocada en carreras relacionadas con la parte agropecuaria, que permitan a los jóvenes aplicar lo aprendido en el entorno rural. (Gf3, estudiantes, 06/10/2016)

Abrirles los ojos a los jóvenes rurales para que caigan en la cuenta de que es lo que tienen en el campo, como estrategia en todos los procesos de enseñanza y a los que son de la parte urbana también mostrarles que el campo es una opción de desarrollo y que todas las personas, sin importar de dónde vengan son iguales. (Ep, estudiante 1, 08/12/2016)

\section{Cayeros plantea la necesidad de considerar a}

Las y los jóvenes [como] sujetos de cambio y de transformación social, que requieren de las oportunidades económicas, sociales y culturales para su desarrollo personal, profesional y ciudadano; por ello, deben ser reconocidos como protagonistas de su propio desarrollo. (2010, p. 1).

En este sentido, Feixa y Oliart (2016, p. 14) enfatizan en la tendencia investigativa de los últimos tiempos al "reconocer la energía creativa, las prácticas críticas y estilos de vida alternativos por los jóvenes, buscando 
enfoques diferentes y la comprensión de las subjetividades juveniles y sus culturas". Por tal razón, concurre la obligación de fijar la mirada en los estudiantes como sujetos activos de los procesos educativos e investigativos para la cualificación de la educación rural.

En relación con lo anterior, una profesora de educación media de la institución reflexiona así:

Los jóvenes hoy en día salen de su entorno, de sus pueblos y sufren transformaciones debido al nuevo mundo al que se enfrentan, esto causa que ellos nieguen o se avergüencen de sus raíces queriendo negar su pasado. El objetivo hoy en día como maestros debe ser crear en los jóvenes y niños esos valores que enriquezcan la identidad por su cultura, costumbres, tradiciones y amor propio, prepararlos para enfrentar los cambios que día a día se presentan sin ofender su personalidad, costumbres y demás aspectos de su cultura. (Nc, profesora 4, 12/01/2017)

En cuanto a las competencias docentes para el desempeño rural eficiente, se destaca la hermenéutica-pedagógica, la cual les exige:

ser competentes para orientar a los estudiantes en la interpretación, diseño e implementación de propuestas de construcción de proyectos de vida que brinden alternativas de mejoramiento y esperanza de una vida cada día más digna. (Ramírez, 2009, p. 135)

Sin embargo, la educación para el sector rural se planea desde las entidades gubernamentales, desconociendo las necesidades y aspiraciones de los jóvenes rurales, por ello, las instituciones educativas tienen la obligación de contextualizar sus currículos. Para circunscribir la educación en el contexto rural, "Se requiere que se tengan en cuenta las necesidades reales de la comunidad a la cual pertenece la zona rural, atendiendo y respondiendo a sus particularidades geográficas, sociales y culturales" (López Rivera, 2015, p. 23).

\section{Conclusiones}

Entre los hallazgos significativos en cuanto al papel de la escuela urbana en la formación de las juventudes rurales, el estudio destaca cómo se desarrolla la socialización entre los jóvenes rurales y urbanos en el ámbito escolar. Los del sector rural procuran ganar espacios dominados por sus pares del pueblo. En estas interacciones resaltan sus valores, el aprecio por su territorio y las labores propias de este contexto.

Otro aspecto importante de resaltar es el de las relaciones de los jóvenes rurales con los docentes, que en su mayoría provienen de la ciudad y desconocen las situaciones particulares de los jóvenes del campo. Sin embargo, los estudiantes procuran manifestar sus condiciones de 
vulnerabilidad, oponerse a todo aquello que los deja en desventaja con respecto a los jóvenes del sector urbano y resistir a las estructuras sociales impuestas por las instituciones, entre ellas la escuela. Además, existe falta de empatía entre los docentes de la escuela urbana y los estudiantes del sector rural al desconocer su contexto sociocultural, debido a las dinámicas mismas de la escuela, en las cuales prevalecen los temas administrativos y el hecho de dictar clases, por encima de las mismas relaciones humanas.

Así las cosas, es importante atender las voces de los estudiantes con la intención de comprender cómo reconfiguran las identidades y los alcances de la escuela en estos procesos, a fin de repensar los currículos y hacer las prácticas educativas pertinentes a los contextos y a las condiciones de los niños, niñas y jóvenes. Esto posibilita descubrir nuevas matrices para pensar la juventud rural, ver a los jóvenes en sus contextos rurales particulares como actores dinámicos capaces de gestionar oportunidades, resistir a condiciones impuestas e innovar en sus realidades complejas. En este sentido, la escuela, según el contexto, se ha clasificado en rural y urbana; existen brechas enormes entre ambas, debido a factores como acceso a tecnologías, recursos bibliográficos, medios de transporte y servicios públicos. Las del sector urbano poco tienen en cuenta las particularidades de los jóvenes rurales. Asimismo, la falta de espacios donde los jóvenes rurales expresen sus saberes artísticos y culturales, las concepciones negativas de los docentes citadinos frente a las posibilidades de transformación y desarrollo en el campo inciden notablemente en la comprensión del impacto de la educación urbana en la construcción de las identidades de las juventudes rurales, razón por la cual los jóvenes reclaman ser tenidos en cuenta en las políticas públicas.

De acuerdo con lo anterior, corresponde a la escuela plantear currículos acordes a los contextos y a las condiciones particulares de los jóvenes rurales, reflexionar con los docentes procedentes de la ciudad acerca de su incidencia en la forma de pensar y actuar de estas poblaciones, a fin de aprender a valorar sus identidades y lograr empoderarlos como sujetos sociales en las transformaciones del sector rural y en la construcción de sociedades justas, democráticas y en paz. Esto requiere promover ambientes adecuados para el desarrollo de una educación que vincule a la familia, la escuela y el contexto sociocultural en el cual se da el acto educativo, de tal manera que los escenarios de exclusión presentes en la vinculación de los jóvenes rurales a los contextos de las escuelas urbanas desaparezcan para dar paso a la promoción de auténticos procesos de inclusión, en los cuales prevalezcan la convivencia fraterna, el respeto a las diferencias y la comunicación efectiva entre los diferentes actores educativos.

Por consiguiente, la investigación permitió reportar las nociones de las creencias y los valores subyacentes en la juventud rural y la manera como la escuela interviene en la reconfiguración de sus identidades. Al respecto,

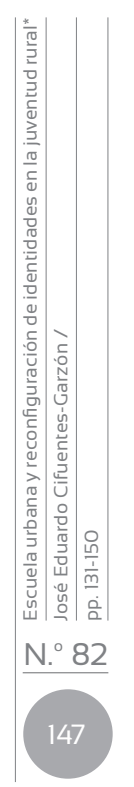


se encontró que las creencias (ideologías, religiosidad y tradiciones) y los valores (hábitos de hogar, principios y vínculos de unión) inciden en la configuración de las identidades (reflejadas en documentos, características propias y la otredad) de los jóvenes rurales. En este sentido, la cultura, las creencias y los valores desde la mirada religiosa y axiológica cobran un significado importante para el asunto investigativo de las identidades en los jóvenes rurales.

De igual manera, resulta pertinente identificar las creencias existentes en los jóvenes rurales y la manera como afectan el mismo empoderamiento de la juventud en los problemas sociales del sector rural. Así las cosas, las actuaciones están relacionadas con la manera como conciben el contexto rural y el acceso a la cultura. Si bien es cierto, el joven rural desempeña un papel fundamental en el ámbito cultural de la región, también cabe destacar su manera de interactuar con los demás y la forma de adaptarse con facilidad a contextos diferentes al suyo. Las concepciones de los jóvenes rurales están ligadas de una u otra forma a las creencias y valores inspirados por los padres y reafirmados por los docentes. Esto fortalece la identidad, forja en ellos comportamientos y descubrimiento de nuevas ideas sobre el rol del joven rural en la sociedad.

Por lo tanto, una labor inaplazable de la escuela es valorar las voces de los jóvenes rurales para identificar las creencias y los valores que guían las actuaciones y que a partir de estos hallazgos puedan orientarse las prácticas educativas. Por ello, existe la necesidad de volver la mirada hacia la formación en valores, que responda al riesgo y la incertidumbre de la sociedad actual. Se requiere una educación enfocada en recuperar la dignidad de la persona humana en los niveles de preescolar, básica, media y universitaria, que trascienda los establecimientos educativos, llegue a la vida del común y toque las maneras de pensar y actuar de la sociedad. La tarea urgente de formar en valores para una sociedad en cambio está vigente en las instituciones educativas tanto rurales como urbanas, desde las particularidades de sus habitantes.

\section{Referencias}

Alvarado, S. (2014). Ampliación de la comprensión de los procesos de configuración de subjetividades políticas de los niños, niñas y jóvenes en Colombia desde una perspectiva alternativa del desarrollo humano: tránsitos y aprendizajes. En S. Alvarado y H. Ospina, Socialización política y configuración de subjetividades: Construcción social de niños, niñas y jóvenes como sujetos políticos. Siglo del Hombre Editores; Universidad de Manizales.

Alzate, F., López, A. y Loaiza, D. (2019). Incidencia del modelo pedagógico en la construcción del proyecto de vida de estudiantes de educación 
media rural. El Ágora USB, 19(1). 95-114. https:// doi.org/10.21500/ 16578031.3494

Anderson, G. (2011). La etnografía de niños y jóvenes en el Estado neoliberal: Una etnografía crítica para nuevos tiempos. En G. Batallán y Neufeldd, M. (coords.), Discusiones sobre infancia y adolescencia: Niños y jóvenes, dentro y fuera de la escuela. Editorial Biblos.

Angrosino, M. (2012). Etnografía y observación participante en investigación cualitativa. Ediciones Morata.

Bustos, A. (2012). La escuela rural. Octaedro Andalucía. Ediciones Mágina.

Cayeros, L. (2010). La construcción de identidades de género entre la juventud rural. [ponencia]. vIII Congreso Latinoamericano de Sociología Rural, Porto de Galinhas.

Chaves, M. (2010). Jóvenes, territorios y complicidades. Una antropología de la juventud urbana. Espacio Editorial.

Estrada, J. A. (2011). Las ambigüedades de la identidad religiosa. En D. Bermejo, La identidad en sociedades plurales. Anthropos Editorial. Universidad de la Rioja.

Etxeberria, X. (2011). El yo moral: Imbricación entre autonomía y alteridad. En D. Bermejo, La identidad en sociedades plurales. Anthropos Editorial.

Feixa, C. y Oliart, P. (2016). Juvenopedia: Mapeo de las juventudes iberoamericanas. Ned Ediciones.

Jurado, C. y Tobasura, I. (2012). Dilema de la juventud en territorios rurales de Colombia: ¿Campo o ciudad? Revista Latinoamericana de Ciencias Sociales, Niñez y Juventud, 10(1), 63-77.

López Rivera, Z. (2015). Educación rural: Una propuesta didáctica para la apropiación social de ciencia, tecnología e innovación desde un enfoque de capacidades para el desarrollo humano. En Cuadernos de Seminario 2. Sociedad y educación en perspectiva rural. Universidad de La Salle.

Lozano, D. (2012). Contribuciones de la educación rural en Colombia: A la construcción social de pequeños municipios y al desarrollo rural. Revista de la Universidad de La Salle, (57), 117-136.

Meseguer, S. (2012). Imaginarios de futuro de la juventud rural: Educación Superior Intercultural en la sierra de Zongolica, Veracruz, México. [Tesis de doctorado]. Universidad de Granada, España.

Oñate, E., Mesurado, B., Rodríguez, L. y Moreno, E. (2018). Práctica religiosa y sentido de vida en adultos jóvenes. Revista de Psicología, 14(27), 57-68.

Parra, R. (1986). Ausencia de futuro: La juventud colombiana. Revista de la Cepal, 29, 81-94.

Parra, R. (2008). El tiempo mestizo, escuela y modernidad en Colombia. En M. C. Laverde, C. E. Valderrama y H. Cubides. Viviendo a toda: Jóvenes, 
territorios culturales y nuevas sensibilidades. DIuc-Universidad Central, Siglo del Hombre Editores.

Parra, R., Parra, F. y Lozano, M. (2006). Tres talleres: Hacia una pedagogía de la investigación etnográfica en la escuela. Convenio Andrés Bello.

Paulín, H. (2016). Sociabilidades juveniles en la escuela: Construcciones de alteridad y luchas por el reconocimiento. En M. Tomasini y H. Paulín (coords.), Jóvenes y escuela: Relatos sobre una relación compleja. Editorial Brujas.

Ramírez, A. (2009). Pedagogía para aprendizajes productivos. Ecoe Ediciones.

Reguillo, R. (2000). Emergencia de culturas juveniles: Estrategias del desencanto. Grupo Editorial Norma.

Torres, O. (2019). Las representaciones de la identidad del estudiante: perspectivas de dos comunidades lingüístico-culturales. Revista Colombiana de Educación, 77. 1(77). https://doi.org/10.17227/rce. num77-7464.

Vommaro, P. (2016). Juventudes y políticas en Argentina y en América Latina: Tendencias, conflictos y desafíos. Grupo Editor Universitario.

Weiss, E. (2012). Los estudiantes como jóvenes: El proceso de subjetivación. Perfiles Educativos, 13(135). 